\title{
COMPARAÇÃO DE MÉTODOS DE AVALIAÇÃO CORPÓREA DE CÃES DA RAÇA LABRADOR E TECKEL
}

\author{
Manara Alves da Silva Leite ${ }^{1}$ \\ Fabiana Ferreira Gama ${ }^{1}$ \\ Diogo Alexandre Tenório Mata ${ }^{1}$ \\ Areane Silva Brito ${ }^{1}$ \\ Pierre Barnabé Escodro ${ }^{1}$ \\ Marcia Kikuyo Notomi²
}

\begin{abstract}
RESUMO
Uma nutrição apropriada é um dos pré-requisitos mais importantes para a saúde e bem-estar animal, e a manutenção do peso ideal pode prevenir inúmeras doenças. Diferentes métodos de avaliação da condição corpórea vêm sendo empregados na medicina veterinária. Apesar da alta reprodutibilidade do escore de condição corpórea (ECC), ainda existem preocupações sobre subjetividade e precisão, e os métodos morfológicos, como o índice de massa corporal canina (IMCC), aparecem como uma alternativa para essa desvantagem, por ser um método quantitativo. Objetivou-se comparar o IMCC e o ECC de cães da raça labrador $(n=30)$ e da raça teckel (daschund) $(\mathrm{n}=20)$, totalizando 50 cães. Informações sobre características dos animais, hábito alimentar e tipo de manejo, também foram analisados quanto à condição corpórea apresentada. A comparação entre os métodos de ECC e IMCC apresentou discrepância de classificação em um elevado número de animais, sugerindo que a característica da morfologia corporal dos cães pode interferir no IMCC, em comparação ao ECC.
\end{abstract}

Palavras-chave: peso corpóreo; obesidade; perda de peso; morfometria

\section{COMPARISON OF BODY ASSESSMENT METHODS FOR LABRADOR AND TECKEL DOGS}

\begin{abstract}
Proper nutrition is one of the most important prerequisites for animal health and welfare, and dogs with optimal weight maintenance can prevent numerous diseases. Different methods of corporeal condition evaluation have been used in veterinary medicine. Although widely used, the body condition score (BCS) is a test influenced by the evaluator experience and a quantitative method, such as the dog body mass index (IMCC), appears as an alternative to this disadvantage. However, the large variation in the body morphology dogs can interfere with their accuracy. The objective was to compare the IMCC and ECC of dogs of the labrador breed $(n=30)$ and the teckel (daschund) breed $(n=20)$, totaling 50 dogs. Information on animal characteristics, eating habits and type of management were also analyzed for the body condition presented. The comparison between the ECC and IMCC methods showed a discrepancy in classification in a high number of animals, suggesting that the characteristic of the dogs' body morphology may interfere in the IMCC, in comparison to the ECC.
\end{abstract}

Keywords: body weight; obesity; weight loss, morphometry

\footnotetext{
${ }^{1}$ Universidade Federal de Alagoas, manaraleite@gmail.com

${ }^{2}$ Universidade Federal de Alagoas. *Correspondência: marcia.notomi@vicosa.ufal.br
}

Leite MAS, Gama FF, Mata DAT, Brito AS, Escodro PB, Notomi MK. Comparação de métodos de avaliação corpórea de cães da raça Labrador e Teckel. Vet. e Zootec. 2021; v28: 001-009. 


\title{
COMPARACIÓN DE MÉTODOS DE EVALUACIÓN CORPORAL PARA PERROS LABRADOR Y TECKEL
}

\begin{abstract}
RESUMEN
Una nutrición adecuada es uno de los requisitos previos más importantes para la salud y el bienestar de los animales, y el mantenimiento del peso ideal del perro puede prevenir numerosas enfermedades. En medicina veterinaria se han utilizado diferentes métodos para evaluar la condición corporal. Aunque ampliamente utilizado, la puntuación de la condición corporal (PCC) es una prueba influenciada por la experiencia del evaluador y los métodos cuantitativos, como el índice de masa corporal canina (IMCC), aparecen como una alternativa a esta desventaja. Sin embargo, la gran variación en la morfología corporal de los perros puede interferir con su precisión. El objetivo fue comparar el IMCC y ECC de perros de la raza labrador $(n=30)$ y la raza teckel (daschund) $(n=20)$, totalizando 50 perros. También se analizó la información sobre las características de los animales, los hábitos de alimentación y el tipo de manejo para determinar la condición corporal. La comparación entre los métodos ECC e IMCC mostró una discrepancia en la clasificación en un gran número de animales, lo que sugiere que la característica de la morfología corporal de los perros puede interferir en el IMCC, en comparación con el ECC.
\end{abstract}

Palabras-claves: salud; peso corporal; obesidad; pérdida de peso, morfometría.

\section{INTRODUÇÃO}

O equilíbrio nutricional é um dos pré-requisitos mais importantes para a saúde e bemestar animal (1). A dieta oferecida pode ajudar no tratamento e na redução do risco às doenças, por outro lado, pode provocá-las com alimentos ou manejos inadequados (2). O equilíbrio energético do organismo está relacionado a uma condição corporal (CC) adequada do animal (1).

A CC é definida como o peso relativo dos diferentes componentes do corpo, como a massa de gordura e a massa magra, com o peso corporal (3), e a sua análise possibilita a identificação de animais que necessitam de dietas controladas (4). Incorporar a avaliação nutricional entre os cuidados de rotina é essencial para a manutenção da saúde dos animais de estimação $(4,5)$. A avaliação da CC exige pouco tempo, baixo custo adicional (5) e possibilita um suporte alimentar adequado, resultando na melhora da resposta terapêutica a enfermidades e ferimentos, assim, promovendo uma redução no tempo de internamento (6). Sua relevância se faz na prevenção de doenças decorrentes da CC alterada e suas consequências (7). É fundamental em enfermidades crônicas, como nefropatias, hepatopatias ou diabetes, subsidiando na introdução de dietas terapêuticas e no acompanhamento nutricional (2). Também é imprescindível em animais saudáveis com exigência metabólica diferenciada, como animais de esporte e trabalho que apresentam um elevado consumo calórico, animais gestantes, em lactação, em crescimento ou idosos, principalmente em condições climáticas de muito calor ou frio $(2,8)$.

Seguindo a tendência humana, a incidência da obesidade vem crescendo em animais de companhia e estudos de diferentes países verificaram uma incidência entre 25 a $40 \%$ de sobrepeso na população de cães, onde diferentes fatores de risco estão envolvidos, dentre eles a genética, a idade, a castração, a redução da atividade física e a mudança de estilo de vida, por estarem vivendo em ambientes menores e com maiores quantidade de energia na dieta $(7,9)$. O reconhecimento precoce da obesidade evita o aparecimento de sérias consequências, como distúrbios articulares e locomotores, endócrinos, cardiorrespiratórios, cutâneos e 
reprodutivos, além do elevado risco à neoplasia, pancreatite, entre outros $(2,7,9)$. Porém, a melhora na sobrevida de animais obesos pode ser observada com o controle sobre o peso corporal em relação a massa magra e gorda, mesmo em cães com a idade avançada (10).

Diferentes métodos de avaliação da CC têm sido propostos, diferindo quanto a sua aplicabilidade, seja na pesquisa, na rotina da clínica veterinária ou para o público leigo, como tutores de animais. Os métodos não invasivos são mais práticos e incluem pesagem, morfometria, pontuação da condição corporal, ultrassonografia e condutância elétrica. (3) Entretanto, existem limitações diante da grande diversidade de raças e das suas peculiaridades anatômicas e morfológicas dependendo da técnica utilizada $(8,11)$.

As técnicas de absorciometria de raios- $x$ de dupla energia (DEXA) e bioimpedância corporal (BIC) avaliam a composição corporal, entretanto de uso ainda restrito aos centros de pesquisa. Já validada em cães (12), a DEXA avalia de forma não invasiva a proporção de tecido ósseo, magro e de gordura corporal $(11,13)$; e a BIC registra a quantidade de água e de gordura presentes no corpo pela passagem de corrente elétrica de baixa intensidade, e estimando a massa magra a partir destes dados $(13,14)$.

O parâmetro mais utilizado para avaliar o estado nutricional em cães é o escore de condição corporal (ECC), onde o avaliador determina um valor a partir da inspeção e palpação do animal, e das características observadas quanto a presença ou ausência de depósitos de gordura, pontas ósseas e massa muscular. Em cães e gatos, os escores de classificação podem variar em até 5, 7, ou 9 dependendo da escala do ECC utilizada, sendo que a de 9 escores foi validada com o DEXA (5). Embora possa ser uma ferramenta útil na gestão do peso corporal em animais de estimação, os sistemas ECC são subjetivos e não distinguem a extensão da obesidade (15).

O peso corporal pode ser usado como um indicador de composição corporal, apresentando uma correlação moderada, mas é limitado pela ampla variação entre raças, faixa etária e sexo. Contudo, é uma importante ferramenta no acompanhamento comparativo de ganho ou perda de peso (3).

$\mathrm{Na}$ morfometria realiza-se uma variedade de mensurações de dobras cutâneas, circunferências e diâmetros ósseos em diferentes segmentos físicos para estimar a composição corporal. Vários estudos avaliaram diferentes locais para correlacionar com a condição corpórea do animal, como altura da cernelha (AC), comprimento corporal (CC), membro pélvico direito (MP), perímetro abdominal (PA), perímetro torácico (PT) e perímetro da coxa (PC) utilizando os dados em equações de predição de massa de gordura e relação óssea (11,15-18). Medidas morfométricas e o índice de gordura corporal pareceram ser mais precisos do que o método ECC de 5 pontos para estimativa do percentual de gordura corporal em cães gordos e obesos (15).

O índice de massa corporal (IMC) é um padrão internacional reconhecido para avaliar o grau de obesidade em humanos (3) e Muller et al. (19) adaptaram o IMC humano para cães, o índice de massa corporal canina (IMCC). O IMC é obtido a partir da fórmula $\mathrm{IMC}=$ peso $(\mathrm{Kg})$ I altura $^{2}(\mathrm{~m})$ e o estudo verificou que a medida da coluna vertebral adicionada ao comprimento do membro pélvico é um parâmetro viável para substituir a altura utilizada em humanos e obter o IMCC. Entretanto, relataram que cães de grande porte $(>25 \mathrm{~kg})$ apresentam um acréscimo de $20 \%$ do IMCC, enquanto que os de pequeno porte (até $10 \mathrm{~kg}$ ) ocorre uma diminuição em $10 \%$ no IMCC, quando comparados com a referência de médio porte. Portanto, os autores sugeriram a utilização dos fatores de correção, ou seja, diminuir $20 \%$ do peso vivo em cães de grande e pequeno porte, aumentar $10 \%$ do índice final para a utilização dos valores de referência.

Aspectos que devem ser considerados na seleção do teste de avaliação da CC são: a facilidade de uso, o baixo custo, não ser invasivo e ter uma boa aceitação por veterinários e clientes. Porém, muitos testes são precisos, mas não exatos, enquanto outros necessitam de 
precisão e exatidão. Atualmente, ainda não existe uma ferramenta perfeita para a análise da $\mathrm{CC}$ em animais de companhia (9). Embora o ECC seja um método eficaz e de uso frequente, na avaliação de gordura corporal pode ser mal interpretada quando realizada por um não profissional, como tutores de animais de estimação, por causa da subjetividade de impressão (18). O IMCC pode ser uma alternativa acessível para a identificação da condição corpórea em cães, acessível tanto a clínica médica, como a tutores de animais. Portanto, objetivou-se comparar o IMCC e o ECC de cães da raça labrador e da raça teckel (daschund)"”.

\section{MATERIAL E MÉTODOS}

O projeto foi aprovado pela comissão de ética animal (CEUA) da Universidade Federal de Alagoas, processo $\mathrm{n}^{\circ}$ 047-15. Foram selecionados para o estudo 50 cães adultos, sendo 30 da raça labrador (19 machos e 11 fêmeas) e 20 da raça teckel (10 machos e 10 fêmeas) com diferentes condições corpóreas, provenientes de tutores particulares. Informações sobre os animais, como peso corporal, sexo, idade, tipo de alimentação recebida (ração comercial, comida caseira ou a associação de ambos) e seu estado reprodutivo (castrado ou não), foram coletadas para análise com os índices da massa corpórea.

Os animais foram avaliados pelo Escore da Condição Corporal (ECC) quanto à presença ou ausência de massa muscular, almofadas de gordura e estrutura óssea visível e/ou palpáveis, classificando-os em: caquético, magro, normal, gordo e obeso (1-5) segundo o descrito por Laflamme $(5,20)$.

A avaliação do IMC canino foi realizada conforme descrito por Müller et al. (19). Para a mensuração considerou-se como pontos de referência a extensão entre a base da nuca e o solo (Figura 1). Iniciou-se a mensuração na articulação atlanto-occipital, seguindo por toda a coluna vertebral sobre o dorso do animal, até a base da cauda (última vértebra sacral), passando imediatamente atrás dos membros posteriores, descendo em uma reta até o solo. Posteriormente foi realizada a pesagem do animal em uma balança digital. Com os dados obtidos, aplicou-se a fórmula de IMC [IMC = peso corporal $(\mathrm{kg})$ x estatura $\left(\mathrm{m}^{2}\right)$ ] classificando-os com a referência que considera uma condição "abaixo do peso" os índices inferiores a 11,7, de "peso ideal" entre 11,8 e 15, "acima do peso" entre 15,1 e 18,6 e "obesos", os IMCC maiores que 18,7. Conforme descrito pelos autores, foi reduzido $20 \%$ do peso vivo dos cães da raça labrador com peso superior a $25 \mathrm{~kg}$ e adicionado $10 \%$ para os cães da raça teckel que apresentavam os pesos inferiores a $10 \mathrm{~kg}$.

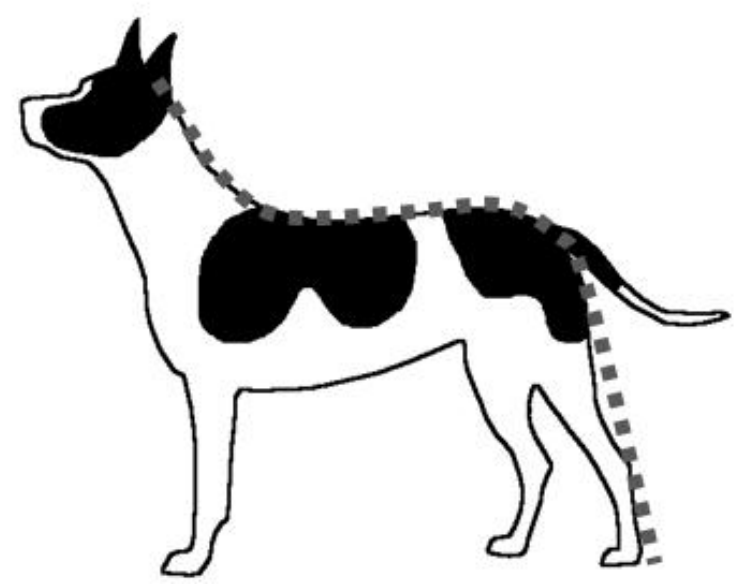

Figura 1. Medida da estatura do cão para o cálculo do índice de massa corporal canino (IMCC) segundo Muller et al. (15).

Leite MAS, Gama FF, Mata DAT, Brito AS, Escodro PB, Notomi MK. Comparação de métodos de avaliação corpórea de cães da raça Labrador e Teckel. Vet. e Zootec. 2021; v28: 001-009. 
Os dados foram tabulados e realizada uma análise da frequência observada para os diferentes escores nos dois diferentes métodos de avaliação de CC e a comparação dos dados, correlacionando o IMCC obtido e a avaliação do ECC.

\section{RESULTADOS}

A idade média dos animais estudados foi de 4,69 anos (valor máximo de 14 anos e mínimo de 1 ano), sendo 3,65 anos a idade média da raça labrador e 6,25 anos da raça teckel. O peso corpóreo médio dos animais do grupo labrador foi de 33,73 $\mathrm{kg}$ (valor mínimo de 23,0 $\mathrm{kg}$; valor máximo $54,0 \mathrm{~kg}$ ), sendo de $34,14 \mathrm{~kg}$ para os machos e $35,14 \mathrm{~kg}$ para as fêmeas; o peso médio dos animais do grupo Teckel foi de $8,54 \mathrm{~kg}$ (valor mínimo $5,0 \mathrm{~kg}$; valor máximo $12,2 \mathrm{~kg}$ ), sendo de $8,96 \mathrm{~kg}$ para os machos e $8,11 \mathrm{~kg}$ para as fêmeas. Com relação ao estado reprodutivo, 26,7\% (8/30) dos labradores eram castrados, seis machos e duas fêmeas e apenas um teckel macho era castrado. Na avaliação do tipo de alimentação, nenhum dos animais era alimentado exclusivamente com comida caseira; dos labradores, 43,3\% (13/30) recebiam uma dieta restrita de ração e 56,7\% (17/30) recebiam ração e outros petiscos, enquanto que para a raça teckel a frequência foi de $45 \%(9 / 20)$ e $55 \%$ (11/20) dos animais que recebiam somente ração e dieta mista, respectivamente. Dos labradores $(n=16)$ e teckel $(n=8)$ considerados acima do peso e obesos pelos de IMCC e ECC, 75\% (12/16) e 87,5\% (7/8), respectivamente, eram alimentados com dieta mista.

$\mathrm{Na}$ avaliação do ECC dos labradores, 10\% (3/30) foram considerados como magros, $23,3 \%$ (7/30) com a condição ideal, 43,3\% (13/30) como gordos e 23,3\% (7/30) como obesos. Os da raça teckel, 5\% (1/20) foram considerados magros, 55\% (11/20) com a condição ideal, $30 \%(6 / 20)$ avaliados como gordos e $10 \%(2 / 20)$ como obesos.

Quanto ao IMCC, após as correções realizadas nos animais com mais de $25 \mathrm{~kg}$ e os com menos de $10 \mathrm{~kg}$, observou-se que a avaliação dos cães labradores variou de 11,78 a 21,13, com a média de 15,71. Segundo os valores de referência de IMCC, 43,3\% (13/30) dos cães apresentavam-se com o peso ideal, 53,3\% (16/30) como gordos e 3,33\% (1/30) obesos. Já a variação na raça teckel foi de 13,56 e 20,95, com média de 17,33, sendo $20 \%$ (4/20) animais em condição ideal e 55\% (11/20) animais gordos e 25\% (5/20) animais obesos. Não foram identificados animais magros ou caquéticos.

Na comparação entre a avaliação ECC e IMCC foi observado que 53\% (16/30) dos labradores foram classificados com o mesmo escore corpóreo entre os dois métodos e dos 47\% (14/30) das avaliações discordantes, em 10\% (3/30) dos casos o IMCC superestimou, isto é, foram avaliados com uma maior condição corpórea; e 37\% (11/30) dos casos subestimou o escore corpóreo quando comparado ao ECC. Nos animais da raça teckel, 55\% $(11 / 20)$ receberam classificação equivalente entre os dois métodos, sendo que em $100 \%$ dos casos discordantes, o IMCC superestimou a condição corpórea quando comparado ao ECC. Não foram observadas diferenças no IMCC entre machos e fêmeas, e animais castrados apresentaram IMCC discretamente maior quando comparados a não castrados. 


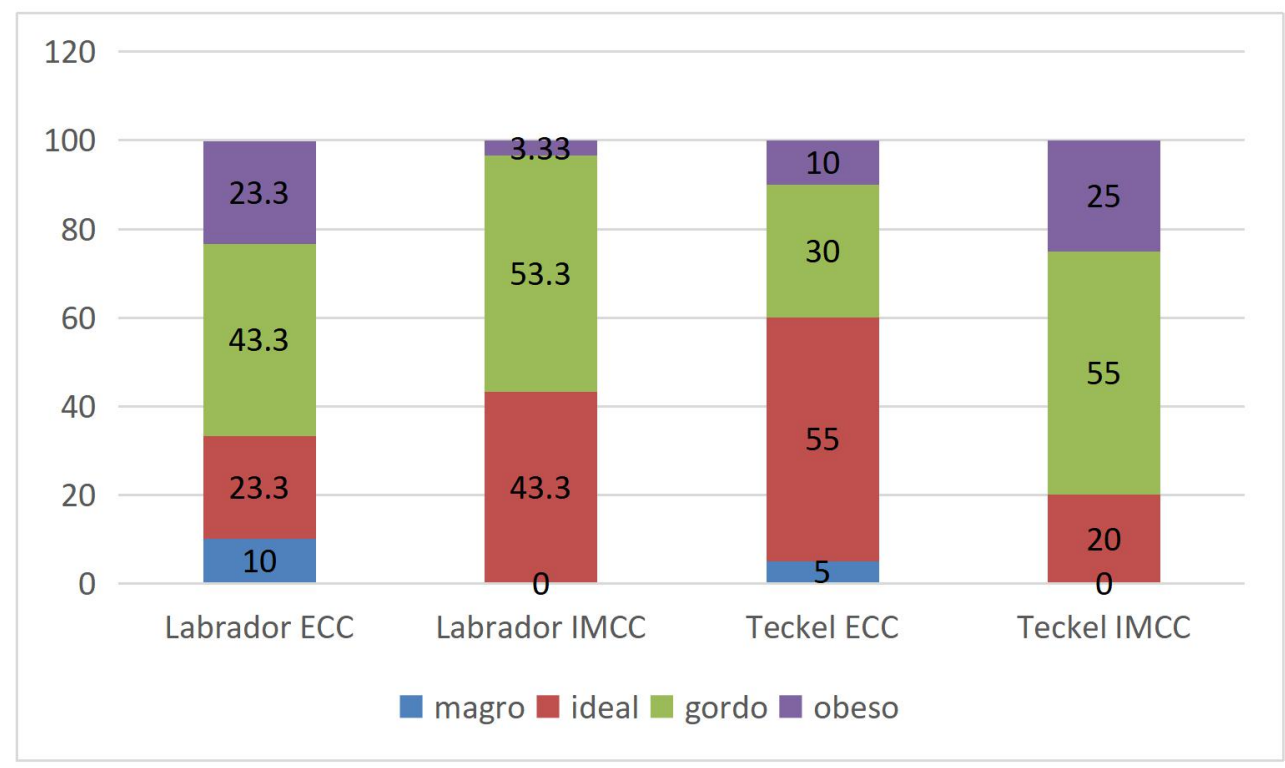

Figura 1. Porcentagem de frequência observada nas diferentes condições corpóreas obtidas. Classificação por ECC e IMCC de cães da raça Labrador e Teckel

\section{DISCUSSÃO}

O estudo avaliou a condição corpórea de cães pelos métodos de ECC e IMCC, observando a concordância na classificação entre os dois métodos em 53\% dos cães da raça labrador e 55\% da raça teckel. Os resultados diferem dos obtidos por Muller et al.(19), porém eles utilizaram cães de diferentes raças e o uso de raças específicas pode ter influenciado nas medidas, uma vez que as características morfológicas raciais podem aparecer de forma mais marcante, como tamanho de focinho, comprimento de tronco e pernas. Uma diversificação de padrões possibilita a diluição das variações da anatomia existente entre as raças, razão pela qual há limitações no uso das diferentes técnicas existentes devido à diversidade de raças e suas peculiaridades $(8,11,15)$.

No caso dos cães da raça teckel, a utilização do fator correção para os animais com menos de $10 \mathrm{~kg}$, agravou a diferença entre os dois métodos. Provavelmente, por sua estrutura morfológica alongada, a aferição do IMCC possa necessitar de um fator de correção diferenciado (11). Estudos morfométricos com bons resultados correspondentes ao DEXA, preferiram não incluir animais de conformação diferenciada ou poucos comuns, excluindo o teckel do experimento (21) ou relatam a dificuldade do uso de medidas morfométricas em algumas raças, como condrodistróficas ou de cabeça grande, entre outras características (15).

Outros fatores que devem ser considerados são a composição do corpo, a distribuição de gordura corporal, massa muscular, ossos e até o aumento volume plasmático provocado por exercícios poderiam afetar os números da equação para o IMCC, como o aumento que pode ocorrer em indivíduos magros com grande musculatura levando a interpretação equivocada de excesso de gordura (19).

A melhor forma de comprovação do IMCC seria a comparação com o DEXA, como o estudo realizado por Jeusette et al. (11) que avaliaram os efeitos da raça / grupo genético na $\mathrm{CC}$ em cães usando DEXA como método de referência e comparando com a bioimpedância, equações morfométricas e ECC, verificando resultados semelhantes de CC. Entretanto, sugerindo que equações morfométricas sejam desenvolvidos por raça ou por grupo de raças para estimar com precisão a composição corporal de várias raças de cães com diferente fundo genético ou morfologia. 
A ausência de diferença no IMCC por gênero, difere da predisposição à obesidade observada em fêmeas $(9,22)$, apesar que a diferença por gênero reduz em animais mais velhos (12). O IMCC elevado dos animais castrados, pode estar relacionado com a diminuição na taxa metabólica após a castração, como também, a uma alteração no comportamento alimentar que conduz ao aumento da ingestão de alimentos e diminuição da atividade física (9). A raça Labrador possui o agravante de apresentar uma predisposição à obesidade $(10,23)$.

Foi observado que a maioria dos animais acima do peso recebiam dietas mistas, que é compatível aos relatos de González et al. (24) que observaram que a combinação de comida caseira com ração comercial constitui-se na principal fonte de alimentação oferecida pelos tutores, sendo esta a mais consumida pelos animais que se apresentam com excesso de peso e/ou obesos.

Identificar o problema da $\mathrm{CC}$ do animal é primordial para estabelecer uma conduta terapêutica, mas muitos tutores não sabem reconhecer que seus animais são obesos ou dos riscos do excesso de peso (25); como agravante, não compreendem os rótulos dos alimentos para animais de estimação (26).

As diretrizes de avaliação nutricional recomendam que as equipes veterinárias avaliem a composição corporal de animais de estimação em cada visita e embora o ECC seja o método indireto mais comum para avaliar o acúmulo de gordura corporal de animais de estimação, ainda existem preocupações sobre subjetividade e precisão deste sistema de pontuação $(2,11,18,27)$.

\section{CONCLUSÃO}

Apesar dos resultados serem coincidentes na maioria dos animais, um elevado número de classificação do IMCC não foi concordante com o ECC nos cães da raça labrador e teckel. Avaliações morfométricas podem ser uma alternativa as limitações do sistema ECC, porém novos estudos devem ser realizados com o IMCC para empregá-lo com mais segurança em cães de diferentes portes e conformações.

\section{REFERÊNCIAS}

1. Guimarães ALN, Tudury EA. Etiologias, consequências e tratamentos de obesidades em cães e gatos - Revisão. Vet Not [Internet]. 2006 [citado 15 Set 2021];12(1):29-41. Disponível em: http://www.seer.ufu.br/index.php/vetnot/article/view/18667

2. Chandler ML, Takashima G. Nutritional concepts for the veterinary practitioner. Vet Clin North Am Small Anim Pract. 2014;44(4):645-66. doi: 10.1016/j.cvsm.2014.03.009.

3. German AJ, Morgan LE. How often do veterinarians assess the bodyweight and body condition of dogs? Vet Rec. 2008;163(17):503-5. doi: 10.1136/vr.163.17.503.

4. Brooks D, Churchill J, Fein K, Linder D, Michel KE, Tudor K, et al. AAHA weight management guidelines for dogs and cats. J Am Anim Hosp Assoc. 2014;50(1):1-11. doi: 10.5326/JAAHA-MS-6331.

5. Baldwin K, Bartges J, Buffington T, Freeman LM, Grabow M, Legred J, et al. AAHA nutritional assessment guidelines for dogs and cats. J Am Anim Hosp Assoc [Internet]. 2010 [citado 15 Set 2021];46(4):285-96. Disponível em: https://www.aaha.org/globalassets/02-guidelines/nutritionalassessment/nutritionalassessmentguidelines.pdf 
6. Ferreira VF, Silva VLD, Ferraz RT. Nutrição clínica de cães hospitalizados: revisão. Pubvet [Internet]. 2017 [citado 15 Set 2021];11(9):901-12. Disponível em: http://pubvet.com.br/artigo/3984/nutriccedilatildeo-cliacutenica-de-catildeeshospitalizados-revisatildeo

7. LaFlamme DP Understanding and managing obesity in dogs and cats. Vet Clin North Am Small Anim Pract. 2006;36(6):1283-305. doi: 10.1016/j.cvsm.2006.08.005.

8. Wakshlag J, Shmalberg J. Nutrition for working and service dogs. Vet Clin North Am Small Anim Pract. 2014;44(4):719-40. doi: 10.1016/j.cvsm.2014.03.008.

9. German AJ. The growing problem of obesity in dogs and cats. J Nutr. 2006; 136(7 Suppl):1940S-6S. doi: 10.1093/jn/136.7.1940S.

10. Penell JC, Morgan DM, Watson P, Carmichael S, Adams VJ. Body weight at 10 years of age and change in body composition between 8 and 10 years of age were related to survival in a longitudinal study of 39 Labrador retriever dogs. Acta Vet Scand [Internet]. 2019 [citado 15 Set 2021];61(1):42. Disponível em: https://actavetscand.biomedcentral.com/articles/10.1186/s13028-019-0477-x

11. Jeusette I, Greco D, Aquino F, Detilleux J, Peterson M, Romano V, et al. Effect of breed on body composition and comparison between various methods to estimate body composition in dogs. Res Vet Sci. 2010;88(2):227-32. doi: 10.1016/j.rvsc.2009.07.009.

12. Grossellin J, Wren JA, Sunderland SJ. Canine obesity - an overview. J Vet Pharmacol Ther. 2007;30 Suppl 1:1-10. doi: 10.1111/j.1365-2885.2007.00863.x.

13. German AJ, Holden SL, Morris PJ, Biourge V. Comparison of a bioimpedance monitor with dual-energy x-ray absorptiometry for noninvasive estimation of percentage body fat in dogs. Am J Vet Res. 2010;71(4):393-8. doi: 10.2460/ajvr.71.4.393.

14. Cintra TCF, Canola JC, Borges NC, Carciofi AC, Vasconcelos RS, Zanatta R. Influência de diferentes tipos de eletrodos sobre os valores da bioimpedância corporal e na estimativa de massa magra $(\mathrm{mm})$ em gatos adultos. Cienc Anim Bras [Internet]. 2010 [citado 15 Set 2021];11(1):149-55. Disponível em: https://www.revistas.ufg.br/vet/article/view/4393

15. Witzel AL, Kirk CA, Henry GA, Toll PW, Brejda JJ, Paetau-Robinson I. Use of a novel morphometric method and body fat index system for estimation of body composition in overweight and obese dogs. J Am Vet Med Assoc. 2014;244(11):1279-84. DOI: 10.2460/javma.244.11.1279.

16. Burkholder WJ, Toll PW. Obesity. In: Hand MS, Thatcher CD, Remillard RL, Roudebush P, Novotny BJ, editors. Small animal clinical nutrition. Topeka: Mark Morris Institute; 2000, p.401-43.

17. Carciofi AC, Gonçalves KNV, Vasconcellos RS, Bazolli RS, Brunetto MA, Prada F. A weight loss protocol and owners participation in the treatment of canine obesity. Cienc Rural. 2005;35(6):1331-8. doi.org/10.1590/S0103-84782005000600016.

Leite MAS, Gama FF, Mata DAT, Brito AS, Escodro PB, Notomi MK. Comparação de métodos de avaliação corpórea de cães da raça Labrador e Teckel. Vet. e Zootec. 2021; v28: 001-009. 
18. Chun JL, Bang HT, Ji SY, Jeong JY, Kim M, Kim B. A simple method to evaluate body condition score to maintain the optimal body weight in dogs. J Anim Sci Technol. 2019;61(6):366-70. doi: 10.5187/jast.2019.61.6.366.

19. Muller DCM, Schossler JE, Pinheiro M. Adaptação do índice de massa corporal humano para cães. Cienc Rural [Internet]. 2008 [citado 15 Set 2021];38(4):1038-43. Disponível em: https://www.scielo.br/pdf/cr/v38n4/a20v38n4.pdf

20. Laflamme DP. Development and validation of a body condition score system for dogs. Canine Pract. 1997;22(1):10-5.

21. Rae LS, Vankan DM, Rand JS, Flickinger EA, Ward LC. Measuring body composition in dogs using multifrequency bioelectrical impedance analysis and dual energy X-ray absorptiometry. Vet J. 2016;212:65-70. doi: 10.1016/j.tvj1.2016.04.007.

22. McGreevy PD, Thomson PC, Pride C, Fawcett A, Grassi T, Jones B. Prevalence of obesity in dogs examined by Australian veterinary practices and the risk factors involved. Vet Rec. 2005;156:695-702. doi: 10.1136/vr.156.22.695.

23. Jericó MM, Albinati JM, Fusco FB. Estudo sobre os hábitos alimentares e as atividades físicas de cães obesos da cidade de São Paulo e seus reflexos no balanço metabólico. Rev Clin Vet [Internet]. 2009 [citado 15 Set 2021];81:54-60. Disponível em: https://revistas.ufpr.br/veterinary/article/view/4012

24. González FHD, Carvalho V, Möller V, Duarte FR. Blood biochemical profile in dogs and cats under different feeding diets. Arch Vet Sci [Internet]. 2003 [citado 15 Set 2021];8(1):23-7. Disponível em: https://revistas.ufpr.br/veterinary/article/view/4012

25. Muñoz-Prieto A, Nielsen LR, Dąbrowski R, Bjørnvad CR, Söder J, Lamy E, et al. European dog owner perceptions of obesity and factors associated with human and canine obesity. Sci Rep. 2018;8(1):13353. doi: 10.1038/s41598-018-31532-0.

26. Yam PS, Naughton G, Butowski CF, Root AL. Inaccurate assessment of canine body condition score, bodyweight, and pet food labels: A potential cause of inaccurate feeding. Vet Sci. 2017;4(2):30. doi: 10.3390/vetsci4020030.

27. Santarossa A, Parr JM, Verbrugghe A. Assessment of canine and feline body composition by veterinary health care teams in Ontario, Canada. Can Vet J [Internet]. 2018 [citado 15 Set 2021];59(12):1280-6. Disponível em: https://www.ncbi.nlm.nih.gov/pmc/articles/PMC6237266/

Recebido em: 04/11/2020 Aceito em: 23/11/2021 